\title{
Contrôle attentionnel lors des régulations. Une autre manière de concevoir l'attention exigée durant les apprentissages
}

\section{Alexandre A.J. Buysse}

De nombreux travaux explorent le travail cognitif qui se déroule hors contrôle attentionnel de l'apprenant, notamment d'un point de vue développemental. Les régulations dont l'apprenant n'a pas conscience semblent jouer un rôle important dans la compréhension et les mises en liens avec la matière précédemment appropriée. Elles ont aussi un rôle fondamental dans la résolution de problèmes. Toutefois, la plupart des démarches didactiques exigent de la part des élèves une concentration lors de la pratique en classe et une explicitation de leurs démarches, ainsi qu'une pratique immédiatement après la transmission du savoir. Sur la base de nos recherches, nous proposons une conceptualisation des liens entre régulation et sources de contrôle des régulations tenant compte de certains éléments connus du fonctionnement du cerveau. Nous abordons aussi la complexité des interactions entre les différentes sources de contrôle dans le cadre du travail en classe.

Nous nous posons la question de l'intégration de la conception biofonctionnelle du cerveau et des dernières avancées en neuroscience aux travaux néopiagétiens sur les régulations. Cet article se veut donc une exploration théorique même s'il se fonde sur nos recherches précédentes portant sur les microgenèses (Buysse, 2008), les sources de régulations (Buysse, 2012) et la réflexivité (Buysse, 2011a, 2011b) ${ }^{1}$.

Le concept de régulation permet d'éclairer comment l'apprenant parvient à trouver de nouvelles solutions, de nouvelles procédures, ou à comprendre de nouvelles notions ${ }^{2}$. En effet, les régulations expliquent "le comment de l'équilibration "(Piaget, 1975, p. 23). Nous pouvons considérer que chaque action, chaque pensée, dépend d'un système qui assure la cohérence du comportement. Quand un sujet rencontre une situation connue, il applique une procédure qui semble lui convenir. Le choix de cette procédure découle d'un système qui permet, par la recherche d'un nouvel équilibre, l'adaptation constante du sujet aux variations de l'environnement.

En effet, on peut considérer qu'une action particulière existait préalablement à son déploiement, en tant que potentiel. Nous parlons alors de schème procédural 
tant qu'il n'est pas contextualisé 3 . Quand ce schème est adapté et actualisé en fonction d'une situation particulière, nous utilisons le terme procédure (SaadaRobert, 1992) ${ }^{4}$. Suivant une vision systémique du fonctionnement cognitif, on peut partir de l'idée qu'à chaque catégorie de situation correspond un système. Lorsqu'une procédure connue est appliquée à une situation nouvelle mais qu'elle ne permet pas d'obtenir le résultat escompté, on considère que le système lié à la procédure entre en déséquilibre. L'observation des régulations permet de cerner comment le système tente de revenir à un nouvel état d'équilibre. Les régulations peuvent par exemple s'observer lorsqu'on prête attention à la manière dont un sujet tente de résoudre un problème. Il déploie des procédures et les ajuste au besoin afin de trouver la manière d'atteindre l'objectif qu'il s'est fixé. Il en va de même lors de l'apprentissage de nouvelles notions si le sujet ne dispose pas des éléments nécessaires pour l'intérioriser. Cette conception peut donc s'étendre à toute élaboration du savoir (Saada-Robert, 1998, 2012; Saada-Robert \& Brun, 1996).

Le processus de régulation consiste ainsi en différentes opérations visant à fixer un objectif à atteindre, orienter l'action vers l'atteinte de l'objectif, contrôler la progression vers le but, confirmer ou modifier la procédure et/ou redéfinir l'objectif (Allal, 2007; Allal \& Saada-Robert, 1992). Ces opérations ne s'enchainent pas nécessairement selon un ordre séquentiel. Ceci est particulièrement vrai si on prend en considération les nombreuses boucles de rétroactions et le traitement parallèle de différentes opérations de régulation, voire de différentes régulations qui s’influencent mutuellement. Chacune de ces opérations dépend également du contrôle du processus de régulation.

Nous pouvons ainsi nous interroger sur la source de contrôle du déroulement d'une régulation. Les régulations dépendantes du sujet s'inscrivent de toute façon dans des régulations liées au système dans lequel il évolue (Volet \& Vauras, 2013). Elles sont, de plus, en interaction étroite avec le système d'activité dans lequel il s'inscrit ou, à tout le moins, du contrôle exercé par le formateur. Les régulations du sujet sont également particulièrement dépendantes du contrôle que les formateurs tentent d'exercer sur les processus d'apprentissage du sujet.

C'est pourquoi, l'autorégulation, vue comme la part contrôlée par le sujet dans les régulations qu'il entreprend, semble indispensable à l'apprentissage, elle ne se conçoit qu'en lien avec des régulations externes au sujet (Allal \& Mottier Lopez, 2007; Allal \& Saada-Robert, 1992, 1998). En effet, le sujet s'inscrit dans un environnement constitué de systèmes en équilibration permanente suite à ses propres actions. Nous reviendrons plus loin sur la complexité de ces interactions.

Le concept de régulation nous permet déjà en soi de considérer que (Allal \& Saada-Robert, 1992, p. 275):

1. Les régulations jouent un rôle important dans le dépassement réflexif des structures, mais surtout elles permettent l'ouverture des structures ${ }^{5}$, c'està-dire la possibilité pour le sujet de construire de nouveaux observables sur l'objet, de prendre conscience et d'identifier les lacunes, perturbations ou contradictions possibles. 
2. Le caractère fondamentalement constructif des régulations permet de considérer la métacognition comme un double mécanisme de construction assurant la formation d'opérations de contrôle (opérations d'anticipation, de contrôle et d'ajustement) et la détermination des constructions des formes explicites des représentations à partir de leurs formes implicites.

Les régulations opérées par le sujet seraient donc fondamentales dans sa résolution de problèmes, dans sa compréhension de la matière et dans l'élaboration d'un sens (Saada-Robert, 2012).

\section{Questionnement}

L'explicitation de ses régulations par le sujet permettrait une amélioration qualitative de celles-ci. Ceci implique donc une prise de conscience par le sujet des régulations à l'œuvre (Piaget, 1974, 1975). Cet impératif de conscience peut néanmoins être questionné, si on prend en compte l'ampleur du fonctionnement cérébral hors de notre contrôle attentionnel. De plus, la compréhension dépendrait d'une part élevée d'autorégulation de la part du sujet. Celle-ci est à nouveau vue le plus souvent comme dépendant d'une capacité de contrôle conscient des régulations de la part du sujet. Là aussi, cette autorégulation du sujet n'existe toutefois pas sans un certain contrôle exercé dans le cours de l'apprentissage ou de l'exécution d'une tâche quelconque, par les formateurs ou l'environnement lui-même, volontairement ou non, explicitement ou non. Nous pouvons donc nous demander dans quelle mesure la conscience du processus de régulation est vraiment indispensable à l'appropriation des nouvelles notions. Nous pouvons poser la question de savoir quels différents contrôles s'exercent sur la régulation de l'apprenant et en lien avec quelles opérations de ses régulations. En effet, nous considérons que toute compréhension repose sur une part au moins d'autorégulation, mais que celle-ci est toujours influencée par des sources externes. Nous devons donc considérer les régulations comme dépendant d'une exécution par l'apprenant durant ses tentatives de compréhension ou durant le déroulement d'une tâche. En effet, le contrôle de l'exécution de celle-ci est, la plupart du temps, partagée et la terminologie considérant les régulations comme des hétérorégulations ou des autorégulations nous semble insuffisante. Nous en venons ainsi à analyser les régulations de l'apprenant sous l'angle de la source de contrôle des régulations.

\section{Les opérations de régulation}

Afin d'élaborer cette notion de contrôle de régulations, nous souhaitons d'abord la considérer selon les opérations de régulation par l'apprenant. Nous voyons, suivant en cela Allal, Rouiller et Saada-Robert (1995), l'autorégulation 
des apprenants comme procédant par trois opérations de nature différente: l'anticipation, le contrôle et l'ajustement:

a. l'anticipation, qui traduit et détermine l'organisation des représentations du sujet en orientations assurant le guidage des processus de production et détermine ce qui devra être contrôlé lors de l'exécution de la tâche et être modifié lors d'un éventuel ajustement:

b. le contrôle (monitoring), qui implique une comparaison entre un état présent en rapport à la tâche et un état-but anticipé:

c. l'ajustement, qui vise la réduction de la distance entre l'état présent et l'état-but par la réorientation des processus de production, impliquant ainsi une modification des représentations et donc de l'anticipation.

Ces opérations ne sont pas purement séquentielles mais interagissent entre elles et modifient leurs résultats grâce à des boucles de rétroaction.

Les connaissances dont l'intégration est visée n'influencent pas la nature profonde de ces différentes opérations. Néanmoins, ces boucles de rétroaction, à différents niveaux, sont particulièrement observables dans le cas de nouveaux apprentissages. Dans ce cas, l'apprentissage exige des régulations qui conduisent à un dépassement et donc à une équilibration dite majorante, en ce qu'elle permet une nouvelle structuration du système. Ces régulations sont alors appelées régulations homéorhésiques - elles font avancer le système plus loin - par opposition aux régulations homéostatiques qui permettent de retrouver l'équilibre du système après un déséquilibre, mais sans progression (Piaget, 1975).

$\mathrm{Si}$ nous tentons de relier les différentes opérations de régulation avec des opérations cognitives plus vastes, notamment dans le cadre d'apprentissages scolaires ou académiques, nous obtenons, en reprenant en grande partie Focant (2003):

1. Planification: suite à une représentation, voire à une analyse de la tâche, la détermination de l'objectif permet d'activer les connaissances disciplinaires, les stratégies qui y ont rapport et les connaissances métacognitives et croyances motivationnelles liées. Elle fixe les procédures et stratégies estimées nécessaires afin d'atteindre l'objectif. Il s'agit d'une phase d'anticipation et de prise de décision.

2. Contrôle: un processus d'auto-surveillance (monitoring) se réalise pour contrôler le cours de l'action en fonction de la planification et évaluer ses résultats en rapport au rapprochement de l'objectif fixé. Ceci se réalise en observant son comportement et en comparant les résultats intermédiaires et finaux obtenus par rapport aux objectifs fixés.

3. Ajustement: en fonction des informations récoltées pendant le contrôle, l'individu décide de maintenir, d'interrompre ou de modifier son action, afin de ramener son comportement en ligne avec l'atteinte de l'objectif. Cette régulation permet également au sujet d'ajuster son répertoire de connaissances et de stratégies stockées en mémoire à long terme en fonction des résultats observés. 
Lorsqu'on analyse la microgenèse des interactions entre un apprenant et un artéfact, nous retrouvons à un niveau d'organisation moins élevé et fonctionnel la même nécessité de régulation que lors des interactions didactiques, ou des tâches, autour d'un objet de savoirs (Saada-Robert, 1992; Saada-Robert \& Brun, 1996).

Ceci étant rappelé, nous pouvons examiner plus en détail en quoi consistent ces opérations.

Nous retenons ainsi que lors de la résolution d'un problème, ou lors d'une intériorisation d'un nouveau savoir, le sujet mobilisera certains processus qui composent les opérations de régulation:

- fixer une finalité ou un but; ces processus permettent ce que nous qualifions d'opération de détermination ${ }^{6}$;

- limiter le cadre de l'action, le système sur lequel s'opèrera la régulation; ceci permet l'opération d'anticipation;

- orienter, planifier, les actions vers le but; ceci permet une opération d'anticipation de laquelle dépend l'appel d'un schème procédural;

- contrôler la progression de l'action vers le but, pendant que le schème est opérationnalisé en tant que procédure adaptée à la situation; ces processus permettent une opération de contrôle; ici l'application de la procédure est comparée au schème et le résultat de cette application est comparé à l'anticipation de l'effet;

- assurer un retour sur l'action (un feedback, une rétroaction); ce processus permet ici une opération de contrôle, qui porte toutefois sur le résultat final obtenu par l'application de la procédure et le compare à l'objectif fixé;

- confirmer ou réorienter la trajectoire de l'action, et/ou redéfinir le but; ceci compose une opération d'ajustement qui permet une restructuration soit par modification du schème et/ou par modification du but.

Sur la base de ce qui précède, nous distinguons les opérations de régulation selon leur caractère:

1. Contrôlant: les différentes opérations de contrôle et d'évaluation à l'œuvre dans les régulations. Elles sont plus ou moins autonomes et sont en général négociées avec le formateur ou déterminées culturellement;

2. Structurant: les différents éléments de sélection des procédures, de détermination des objectifs et d'anticipation du résultat visé. Un formateur peut apporter un éclairage sur les logiques et concepts menant à ces processus. Ceci aide le sujet à comprendre, à trouver des solutions face à des problèmes inédits et donc à intérioriser de nouveaux savoirs.

Ce caractère contrôlant ou structurant implique que les informations nécessaires pour ces opérations soient de nature différente. Ceci explique qu'on peut classifier la réflexivité selon sa contribution aux opérations de régulation, en réflexivité contrôlante et réflexivité structurante (Buysse, 2009). La réflexivité est ainsi vue comme ce qui nourrit le processus de régulation, tant par sa part de réfléchissement que par sa part de réflexion (Piaget, 1977). 
Nous obtenons donc une conceptualisation des régulations tenant compte des opérations de régulation et du caractère de l'opération (tableau 1), quelles que soient les influences exercées sur celles-ci.

\section{Tableau 1: opérations, caractère}

\begin{tabular}{|c|c|c|}
\hline Opérations & Processus composant l'opération & Caractère \\
\hline Détermination & $\begin{array}{l}\text { Fixer un but, un objectif } \\
\text { (résultant éventuellement de l'ajustement à la fin de la } \\
\text { boucle de régulation précédente) }\end{array}$ & structurant \\
\hline \multirow[t]{2}{*}{ Anticipation } & $\begin{array}{l}\text { Limiter l'étendue de l'action } \\
\text { (résultant éventuellement de l'ajustement à la fin de la } \\
\text { boucle de régulation précédente) }\end{array}$ & contrôlant \\
\hline & $\begin{array}{l}\text { Orienter (planifier) l'action vers le but } \\
\text { (résultant éventuellement de l'ajustement à la fin de la } \\
\text { boucle de régulation précédente) }\end{array}$ & structurant \\
\hline \multirow[t]{2}{*}{$\begin{array}{l}\text { Contrôle } \\
\text { (monitoring) }\end{array}$} & $\begin{array}{l}\text { Contrôler la progression de l'action } \\
\text { selon la conformité avec la planification }\end{array}$ & contrôlant \\
\hline & $\begin{array}{l}\text { Assurer un retour sur l'action (un feedback, une rétroaction) } \\
\text { Contrôle en tant qu'évaluation progressive du résultat des } \\
\text { actions entreprises afin d'atteindre l'objectif }\end{array}$ & contrôlant \\
\hline Ajustement & $\begin{array}{l}\text { Confirmer ou réorienter l'action, et/ou redéfinir le but, ce } \\
\text { qui mène ainsi à une nouvelle anticipation ou détermination }\end{array}$ & structurant \\
\hline
\end{tabular}

Les régulations se situent en effet dans un environnement complexe et peuvent autant relever d'opérations inhérentes au sujet - autorégulation - qu'être dépendantes de l'activité, provoquées par un formateur, des pairs, la situation ou des outils (Allal \& Saada-Robert, 1992).

\section{Sources de contrôle des régulations}

En effet, l'apprenant est rarement seul face à une tâche et les différents processus inhérents aux opérations de régulation peuvent être influencés par des formateurs, des pairs ou les outils à disposition. L'autorégulation dépend donc également de régulations autres que celles de l'apprenant. Ces influences externes s'exercent ainsi sur les processus à caractère contrôlant ou structurant des opérations de régulation de l'apprenant.

S'il y ainsi des régulations plus ou moins influencées de l'extérieur, voire relevant totalement de régulations externes à l'apprenant, il y a également des niveaux de complexité exigeant différentes régulations de la part de l'apprenant lui-même.

En effet, la complexité de la résolution de problème ou la complexité du savoir à s'approprier est telle qu'elle repose sur plusieurs régulations de la part de l'apprenant. Parmi celles-ci, certaines régulations peuvent être maitrisées depuis longtemps alors que d'autres posent encore problème. Par exemple, dans le cas 
de la rédaction d'une lettre, nous pouvons avoir des régulations portant sur la formulation du propos, l'orthographe d'usage à employer pour écrire les mots, l'orthographe grammaticale à respecter, la formation des lettres composant chacun des mots. Chacun de ces éléments de la tâche de rédaction d'une lettre demande des régulations particulières.

Si l'apprenant devait dédier simultanément son attention à toutes les régulations nécessaires, la tâche deviendrait impossible à gérer. On constate ainsi que certaines composantes de la tâche, sous-tâches déjà maitrisées, peuvent ne faire l'objet d'aucune attention. En effet, la plupart du temps, certains éléments sont déjà familiers, même s'ils demandent des régulations, alors que d'autres sont entièrement nouveaux. Dans notre exemple, la formation des lettres n'exigerait que peu ou pas d'attention de la part d'un adulte lettré, alors que l'orthographe grammaticale ou la formulation du propos pourrait, au contraire, exiger toute son attention.

On considère ainsi qu'un nombre de sous-tâches dont l'accomplissement est indispensable font l'objet de régulations automatisées (Allal, 2007). Quand la résolution d'un problème entraine une régulation homéorhésique - qui résulte en l'élaboration de nouveaux schèmes et/ou l'appropriation de nouvelles connaissances -, cette régulation homéorhésique est le plus souvent coordonnée avec des régulations plus homéostatiques - permettant le rétablir l'équilibre sans progression notoire - d'autres composantes de la tâche. L'apprenant doit en effet mettre en œuvre et mettre en relation des connaissances préexistantes pour atteindre un nouvel objectif.

Qu'il s'agisse de régulations explicitées ou non, nous considérons que les opérations fondamentales de l'autorégulation (détermination, anticipation, contrôle, ajustement) se déroulent dans tous les cas. L'autorégulation a lieu sous différentes formes allant, sans discontinuer, de régulations implicites, automatisées ou non, aux régulations explicites volontaires (Allal, Rouiller \& Saada-Robert, 1995).

Tel que mentionné précédemment, "pour des tâches relativement complexes, exigeant la coordination de plusieurs processus de production, la régulation de certains processus se déroule de manière automatique, tandis que la régulation d'autres processus exige la gestion active et intentionnelle des ressources cognitives à disposition» (Allal \& Saada-Robert, 1992, p. 273). Les régulations jouent ainsi un rôle majeur dans l'acquisition des connaissances, mais elles opèrent également une reconstruction contextuelle lors des transferts, c'est-à-dire lors de l'utilisation de connaissances automatisées dans de nouveaux contextes.

Nous pouvons donc nous représenter les régulations et leur contrôle comme un système composé de sous-systèmes de régulations et de contrôle, la plupart automatisées. Le système de contrôle attentionnel central reprend, de temps à autre, une gestion active afin de dédier toute l'attention à la remédiation d'un problème plus important rencontré par un des sous-systèmes. Comme dans le cas d'une hésitation quant à quel temps utiliser lors de l'écriture d'une partie 
d'un texte.

Cette représentation du contrôle attentionnel admet ainsi l'abandon $\mathrm{du}$ contrôle de certaines régulations par le contrôle attentionnel central, lors de l'exécution de tâches complexes. Il y a ainsi délégation du contrôle attentionnel à un sous-système de régulation, pour reprendre une terminologie cognitiviste. Suite à cette délégation, le sujet n’a plus immédiatement conscience des processus en cours lors de la régulation effectuée par un sous-système. Cette délégation à un sous-système est en vigueur tant que le contrôle du déroulement de la régulation au sein de celui-ci ne signale pas un problème. Si ceci se produit, la tâche est portée à la conscience de l'apprenant et devient un problème à résoudre en soi auquel il consacre son attention.

Ceci nous conduit à considérer les quatre degrés d'explicitation des régulations, à prendre en compte en raison de «leur continuité fondamentale et de leurs articulations fonctionnelles» (Allal et Saada-Robert, 1992, p. 277):

1. les régulations implicites, intégrées au fonctionnement cognitif, qui ne sont pas portées à la conscience du sujet;

2. les régulations accessibles au niveau attentionnel et explicitables, c'est-à-dire celles que le sujet serait capable d'expliciter si on lui demande de le faire, ou si les exigences de la tâche provoquent un effort d'explicitation;

3. les régulations explicitées, sur lesquelles il opère avec intentionnalité et qu'il communique à autrui;

4. les régulations instrumentées qui s'appuient sur un support externe à la pensée du sujet et qui, de ce fait, peuvent conférer en retour aux processus mentaux de régulation une puissance - durabilité temporelle, souplesse, généralité - accrue. L'instrumentation des régulations peut se baser sur un support que le sujet a produit pour lui-même ou venant d'autrui pour autant que l'apprenant se soit approprié l'outil en question.

Le sujet n'a ainsi pas toujours le besoin ou la capacité d'expliciter ses régulations, car elles peuvent être tellement maitrisées, intégrées au fonctionnement cognitif, qu'elles ne requièrent plus son attention. Elles peuvent également reposer sur des outils intégrés à son fonctionnement et, là aussi, sur une éventuelle explicitation. Cette explicitation repose entièrement sur un contrôle attentionnel. Cette conscience du déroulement peut certes être un retour sur la régulation après coup, mais sous-entend que celle-ci est re-traçable. Dans le modèle proposé jusqu'ici les seules régulations implicites, intégrées au fonctionnement cognitif, sont celles qui sont tellement familières qu'elles sont automatisées. Il ne s'agit aucunement de nouveauté. Cela sous-entend également que toutes les opérations de la régulation sont soit implicites, soit explicites. Nous proposons donc d'approfondir la notion de contrôle des régulations selon deux axes: celui du contrôle attentionnel et celui des opérations de la régulation.

Selon Iran-Nejad (1990), le fonctionnement du cerveau humain ne repose pas uniquement sur le contrôle attentionnel. Bien au contraire: la plus grande 
activité a lieu en dehors de notre contrôle conscient. En dehors des régulations automatiques non-problématiques se déroulant en parallèle de la plupart des tâches (Allal, 2007; Allal \& Saada-Robert, 1992), toute régulation nécessite de dédier de l'attention au processus de régulation. Si cela dépendait du contrôle attentionnel central nous constaterions systématiquement un phénomène de goulot d'étranglement ralentissant, voire interférant avec, le processus de régulation. En effet, le contrôle attentionnel central opère de façon séquentielle, créant l'illusion d'un traitement simultané grâce à la sollicitation de la mémoire à court terme et de l'alternance dans le passage du contrôle attentionnel d'une tâche à l'autre (Cohen \& Schooler, 2013; Yeung, Nystrom, Aronson, \& Cohen, 2006). On ne peut ainsi accorder, au même instant précis, sa pleine attention qu'à une régulation à la fois. La tentative de contrôler consciemment et simultanément le déroulement de plusieurs régulations au même moment engendre un certain coût cognitif, lié au fait que le contrôle attentionnel central alterne rapidement d'une régulation à l'autre, résultant en un ralentissement de la résolution de problèmes ou en des erreurs de jugement. La surcharge cognitive engendrée par ces traitements simultanés peuvent même affecter, la capacité ultérieure de faire des choix ${ }^{7}$ (Vohs et al., 2014). Nous pouvons simplifier cela en nous représentant que l'attribution de l'attention est séquentielle et ne peut donc s'exercer que sur un système de régulation à la fois. Il en résulte que, si nous devions avoir conscience de toutes nos régulations, nous ne pourrions traiter qu'un seul problème à la fois.

Toutefois, Iran-Nejad (1990) observe que de nombreux sujets trouvent des solutions à des problèmes alors qu'ils sont en train de procéder à une régulation portant sur une autre tâche. Ceci s'explique par le fait que notre capacité attentionnelle est séquentielle et réduite. En effet, notre système attentionnel central ${ }^{8}$ nous permet de traiter un seul élément d'information à la fois et est notamment sensible au coût de l'effort de concentration (Iran-Nejad \& Zengaro, 2013; Kurzban, Duckworth, Kable, \& Myers, 2013). Il est toutefois capable d'alterner rapidement, ce qui nous donne l'illusion d'un traitement simultané. Certains éléments, totalement intégrés à notre fonctionnement, ne nécessitent pas toute notre attention. Cela ne suffit pas toutefois à décharger la pression exercée sur notre contrôle attentionnel. Imaginons simplement que nous devrions tout comprendre à l'instant même où cela nous est expliqué et qu'une compréhension ultérieure ne serait possible qu'en y accordant toute notre attention: ce serait impossible.

Il existe toutefois une explication possible reposant sur une conception biofonctionnelle du cerveau et sur le fonctionnement en alternance entre le contrôle attentionnel central et une délégation du contrôle attentionnel à des sous-systèmes du cerveau ${ }^{9}$ (Iran-Nejad, 1990). On peut ainsi tenter de distinguer les sources du contrôle attentionnel exercé sur le processus d'autorégulation (Buysse, 2012; Iran-Nejad, 1990), tout en tenant compte du fait que la plupart des régulations font l'objet d'un contrôle partagé avec d'autres personnes, 
notamment dans les contextes d'apprentissage. Nous parlerons donc d'une régulation ou d'une partie des opérations de régulation dépendant entièrement ou partiellement d'une source de contrôle attentionnel:

1. directe, pour la part ayant lieu sous contrôle externe à l'apprenant, par des instruments, un formateur, etc.;

2. automatisée, pour la part dépendant de l'apprenant mais n'exigeant pas un contrôle attentionnel de sa part car ne présentant aucune difficulté;

3. active, pour la part sous le contrôle attentionnel de l'apprenant, car présentant en général des difficultés; impliquant un traitement séquentiel de l'information, sous contrôle exécutif, avec une limite stricte de capacité mais la possibilité d'être relayée par la mobilisation conjointe des processus de régulation automatique;

4. dynamique, dépendant de l'apprenant mais hors de son contrôle attentionnel central, lors d'un processus de régulation ou d'une part de processus de régulation qui est souvent différé, qui reconceptualise, innove, tisse des liens entre ce qui est appris et les connaissances de l'apprenant; reposant sur un traitement parallèle d'informations à partir de «multiples ressources locales» et sans limitation stricte de capacité.

Notons que nous pouvons considérer qu'une régulation est soit pilotée entièrement par une unique source de contrôle, directe, automatisée, active ou dynamique, ou qu'elle est l'objet d'un contrôle différent selon les processus composant les opérations (voir tableau 1). Lors de l'appropriation du savoir, ces différents contrôles se complètent et s'exercent sur des opérations différentes du processus de régulation et sont priorisées lors de certaines occasions.

D'une part, le contrôle actif ${ }^{10}$ peut se définir comme un contrôle attentionnel de la part de l'apprenant. Le sujet dédie son attention à ce qu'il fait ou à ce qu'il examine. Il s'agit ici de la conception classique d'autorégulation non-automatisée. En effet, le sujet fait preuve d'un contrôle actif dans la phase d'intériorisation d'un savoir ou lorsqu'il recherche une solution. Les opérations de régulation impliquées étant multiples, une partie des tâches non-problématiques sont déléguées à des sous-processus de régulations automatisées, donc implicites. Le sujet peut ainsi dédier son attention au processus problématique. Ce contrôle actif, objet de la plupart des études métacognitives, est caractérisé par des régulations volontaires le plus souvent explicites et instrumentées (Allal \& Saada-Robert, 1992).

D'autre part, le contrôle dynamique est un fonctionnement mental spontané, pendant lequel les processus de régulation seraient assurés par un ensemble de sous-systèmes de régulation agissant en tant que composantes non-exécutives du système. Le contrôle de la régulation est dans ce cas assuré au niveau des composantes et le sujet n'y dédie donc pas son attention. Celle-ci est donc libérée pour d'autres régulations exigeant un contrôle actif. Quand le sujet ne parvient pas à trouver immédiatement une solution, il semble déléguer une partie des processus de régulation vers une régulation dynamique opérant en dehors de son 
contrôle attentionnel (Iran-Nejad \& Chissom, 1992). Des liens seraient ainsi établis entre des savoirs existants et les nouvelles données, sans que le sujet puisse expliciter l'ensemble de ce processus. Ces régulations sous contrôle dynamique semblent également permettre l'intégration progressive des nouvelles découvertes résultant des régulations actives de l'apprenant.

Il convient toutefois de distinguer la régulation automatisée, basée sur des compétences acquises, qui est très stable et difficile à modifier, de la régulation dynamique qui, quoique stable, est très facile à modifier. Toutes les deux opèrent au niveau des sous-systèmes de régulation mais le contrôle interne dynamique, par des sous-systèmes de régulation, reste profondément heuristique. Ce contrôle dynamique permet ainsi, après une éventuelle délégation intentionnelle, une certaine liberté de réorganisation des sous-systèmes selon les besoins engendrés par des changements de contexte (Iran-Nejad \& Chissom, 1992).

La régulation active, vu que le problème est amené au niveau attentionnel, agit ainsi comme un organisateur des processus non attentionnels à l'œuvre dans la régulation dynamique. Ces processus non attentionnels ${ }^{11}$ sont conditionnés par les «actes préalables de connaissance rationnelle, de compréhension, de reconnaissance, d'association» (Vygotski, 1925/2005, p. 357) et dépendent donc de la direction que l'on donne aux régulations actives.

Les régulations ressortant de l'une ou l'autre source peuvent se transformer en cours de fonctionnement et, de ce fait, être sources de construction de nouvelles compétences. On peut aisément concevoir que la régulation dynamique est à l'œuvre quand on ne pense pas consciemment à un problème, on n'y dédie pas notre attention, on fait même complètement autre chose, voire on laisse passer des jours entiers sans s'en préoccuper, mais que pourtant, après ce temps d'incubation $^{12}$, on trouve, hors contexte, la solution. Par contre, on peut être intrigué par le fait que la régulation dynamique puisse être à l'œuvre dans les restructurations suite à un nouvel apprentissage.

Dans la conception de la rééquilibration des systèmes et de la reconstruction à un palier supérieur, en tant que moteur développemental, l'équilibration d'un système ou cycle a un effet sur les autres équilibrations effectuées précédemment. Cet effet échappe toutefois au contrôle attentionnel du sujet. En effet, on ne saurait dire que l'on travaille, au niveau attentionnel, tous les réajustements qui résulteraient d'une nouvelle conception. Il en résulte que ces réélaborations des systèmes à un niveau supérieur sont bel et bien de l'ordre des régulations dynamiques. Si l'apprentissage a un caractère en grande partie de régulation active, le développement serait presque exclusivement de l'ordre de la régulation dynamique.

Un changement constructif pourrait ainsi se produire en l'absence d'une régulation active parce que l'attention est déléguée à des sous-systèmes de régulation. Cette délégation d'attention semble s'effectuer de manière implicite ou sous contrôle exécutif direct, explicite. Dans ce cas, la délégation n'est pas nécessairement immédiate. Ces composantes, ou sous-systèmes, peuvent ainsi 
réguler les processus d'apprentissages locaux et des changements de niveau local sans exiger d'attention immédiate. C'est ainsi que la délégation d'attention, et non l'attention elle-même, se retrouve au centre des processus de régulation.

Il y a, particulièrement dans les cas d'un effort heuristique ${ }^{13}$ important, délégation de la régulation, non seulement des sous-tâches non-problématiques à des sous-systèmes de régulation sous contrôle automatisé, mais également fractionnement du système à réguler en différents sous-systèmes. Certains de ceux-ci se feraient sous contrôle dynamique, implicite et hors contrôle attentionnel de l'apprenant. Ces régulations sous contrôle dynamique établiraient des liens entre des savoirs existants et les nouvelles données. Ces sous-systèmes tentent ainsi également d'intégrer progressivement les nouvelles découvertes résultant des régulations actives de l'apprenant. C'est ainsi que la régulation dynamique se retrouve au centre de la reconceptualisation et de la restructuration des schèmes existants. Toutefois, les résultats des régulations dynamiques par les sous-systèmes sont gérés par des régulations actives qui évaluent, au niveau attentionnel, leur adéquation par rapport aux enjeux d'origine.

L'apprentissage de schèmes complexes n'est pas possible uniquement grâce à l'interrelation entre des régulations actives et des régulations automatisées, mais s'opère à travers une reconstruction interne régulée par deux sources différentes de contrôle de l'autorégulation: la régulation active et la régulation dynamique (Iran-Nejad, 1990). Selon sa vision, l'apprentissage d'un schème complexe est une reconceptualisation créative d'un savoir grâce à une régulation dynamique et la plus grande partie de l'apprentissage se déroule en dehors du goulot d'étranglement que constitue le contrôle attentionnel central - conscient - mobilisé par une régulation active.

Hormis son caractère novateur, moteur probable de l'innovation, la régulation dynamique semble donc être l'élément qui permet le développement. Hagmann et al. (Hagmann et al., 2008) ont établi que, durant l'absence d'un contrôle attentionnel actif, une activité intense d'interconnexion entre des zones spécialisées du cortex a lieu. Ils émettent l'hypothèse que ceci joue un rôle important dans l'intégration fonctionnelle. Suite aux résultats de l'imagerie cérébrale, ils suggèrent également une source possible de ce contrôle et établissent un lien entre l'état de repos et la restructuration:

Des régions structurellement séparées et fonctionnellement spécialisées du cortex cérébral humain sont interconnectées par un réseau dense de voies axonales cortico-corticales ${ }^{14}$. [...] Une analyse des réseaux structuraux cérébraux à grande échelle révèle un noyau structurel à l'intérieur des cortex médial et pariétal, ainsi que différents noyaux temporaux et frontaux. [... Ces régions] constituent des noyaux de connexion qui relient tous les principaux modules structurels. [...] Nous avons constaté une correspondance significative entre la connectivité structurelle et la connectivité fonctionnelle à l'état de repos chez les mêmes participants. La centralité spatiale et topologique au sein du cortex suggère un rôle important dans l'intégration fonctionnelle. 
(Hagmann et al., 2008, p. 1479)

Ce qui retient également notre intérêt, c'est le fait que, d'après leurs recherches, ce centre de coordination est non seulement central au niveau de son emplacement mais également proche de certains éléments du réseau neuronal par défaut, soulignant, à notre avis, son caractère intimement lié au niveau naturel du développement: "Sa correspondance anatomique avec des régions d'activité métabolique élevée et avec certains éléments du réseau par défaut suggèrent que le centre peut être une base structurelle importante dans la formation de dynamiques cérébrales à large échelle» (Hagmann et al., 2008, p. 1489).

Le lien entre différentes zones spécialisées du cerveau serait dès lors dépendant de connexions se construisant en dehors du contrôle attentionnel.

Une comparaison de l'imagerie de diffusion et d'imagerie fonctionnelle en état de repos révèle une relation étroite entre les connections structurelles et fonctionnelles, y compris pour des régions formant le noyau structurel [«structural core»]. [...] les données de l'imagerie anatomique et fonctionnelle suggèrent un rôle important pour le noyau dans l'intégration de l'information cérébrale. (Hagmann et al., 2008, p. 1479)

Ceci nous mène à concevoir la régulation homéorhésique qui mène à l'équilibration majorante en tant que processus de développement interne au sujet, sous l'angle de sa qualité restructurante, comme largement dépendante de fonctionnements non dirigés par notre attention. La conséquence est qu'il convient de concevoir l'enseignement comme apport externe au sujet, exigeant entre autres un contrôle actif de sa part, donnant les bases permettant cette réorganisation ultérieure.

\section{Variation dans le contrôle des processus}

Nous pouvons repérer l'ampleur du recours à l'un ou l'autre contrôle attentionnel des régulations - direct, actif, dynamique ou automatisé - soit par l'analyse des microgenèses en tenant compte des régulations différées et de leur déploiement (Buysse, 2008), soit par une explicitation par les apprenants sur la base d'un questionnaire, le DALI-R (Iran-Nejad \& Chissom, 1992). Le recours préférentiel à l'un ou l'autre contrôle de l'autorégulation évolue à travers le temps mais dépend également des situations d'enseignement/apprentissage.

Pour l'enfant, au début de son développement dans un environnement stimulant, l'apprentissage semble régulé par un fonctionnement simultané et un contrôle dynamique. Ce contrôle dynamique semble plus présent qu'un contrôle actif reflétant une concentration soutenue dans le temps. Le contrôle actif émerge progressivement et des différences entre les apprenants quant à l'autorégulation commencent à apparaitre selon l'interaction entre les sources du contrôle (externe, dynamique ou active), les processus d'apprentissage et les 
savoirs connus. Si la régulation dynamique semble ainsi présente dès la naissance, relevant selon Iran-Nejad (1990) d'un fonctionnement biologique spontané, les apprentissages et particulièrement la scolarisation amènent une dominance progressive de la régulation active, largement culturelle.

Ceci rejoint les résultats dans la recherche sur le développement du cerveau du bébé (Gopnik, 2010). Des liens se créent constamment, même dans les moments passifs du bébé, et ces activations neuronales sont visibles à l'IRM. Il se pourrait qu'il y ait ainsi une ouverture beaucoup plus grande aux réorganisations et aux apprentissages implicites que dans le cas d'un contrôle attentionnel actif.

Néanmoins, à mesure que les enfants sont amenés à dépendre de régulations actives, qui ne sont souvent pas entrainées ou mal entrainées dans le cadre scolaire, ils ne se laissent plus aller à des régulations dynamiques (Iran-Nejad \& Chissom, 1992). Ils réduisent ainsi leur potentiel. Ils risquent de finir par appliquer des méthodes dont la découverte est guidée, au lieu de créer des solutions originales. En effet, l'insistance sur l'explicitation avant ou pendant l'action demande une concentration permanente de l'apprenant. Cet appel au contrôle actif afin de répondre au besoin de suivi par le formateur ne permet pas à l'apprenant d'avoir recours à des régulations dynamiques, augmentant les risques de surcharge cognitive. Certains apprenants finissent par dépendre d'autant plus du formateur, faute de pouvoir avoir recours aux processus de contrôles spontanés et faute d'une formation portant sur l'organisation préalable à la régulation permettant d'avoir recours plus largement au contrôle actif ${ }^{15}$.

Nous voyons ainsi se dessiner un parallèle avec les écrits sur le développement de la créativité (Vartanian, 2013). En effet les conditions favorables à la créativité rejoignent celles favorables à un contrôle dynamique de régulations (Gregg \& Carver Sekeres, 2008). Il semblerait que différents contextes peuvent stimuler la créativité et notamment le fait d'amener les élèves et étudiants à poser des problèmes et à développer une pensée critique. Néanmoins, une trop grande intervention externe, contrôle externe, motivation extrinsèque, etc., particulièrement dans le cadre scolaire, pourraient réduire le recours à la créativité (Boden, 2004; Fasko, 2001; Lubart \& Georgsdottir, 2004; Runco, 2003; Sawyer, 2012; Sawyer et al., 2003).

La détermination et la prise en compte des facteurs favorisant la régulation dynamique nous semblent d'autant plus importants que la réussite scolaire apparaît liée à la capacité de combiner un contrôle actif avec un contrôle dynamique (Iran-Nejad, 1990; Iran-Nejad \& Chissom, 1992). Shapiro et Livingston (2000) trouvent en effet que les apprenants démontrant un style de régulation dynamique réussissent mieux que ceux pratiquant avant tout une régulation active. De plus, il semblerait que le fonctionnement du cerveau invite à un traitement global avant de pratiquer des éléments isolés, voire même avant de mettre des éléments en association les uns avec les autres. Le raisonnement global, contrôlé dynamiquement et hors de notre contrôle attentionnel, serait ainsi à la base de notre fonctionnement mental. Ceci explique que notre capacité 
attentionnelle n'a pas besoin d'être autant développée et que le coût énorme de celle-ci peut être contourné par notre fonctionnement dynamique. Ceci permettrait d'expliquer que certaines décisions soient prises avant même que nous n'en soyons conscients (Soon, Brass, Heinze, \& Haynes, 2008).

\section{Une conceptualisation du contrôle des régulations}

Nous proposons une conceptualisation du contrôle des régulations en fonction des opérations de régulation et du degré d'intériorisation du savoir. En effet, la maîtrise du savoir peut s'observer dans les explicitations par les apprenants. Les caractéristiques de leurs explicitations reflètent différents niveaux d'intériorisation du savoir (Buysse \& Vanhulle, 2009; Valsiner, 1997):

- l'acquisition du savoir, vue comme la phase d'apprentissage et ne rendant pas compte d'une compréhension en profondeur;

- l'appropriation du savoir, vue comme la compréhension du savoir en fonction des concepts qui sont véhiculés avec le savoir, donc de références objectives;

- la subjectivation, vue comme la compréhension du savoir en fonction des éléments déjà intériorisés par le sujet, donc de références subjectives;

- la maîtrise, vue comme un degré développemental d'intériorisation incluant l'intégration à la fois des références subjectives et objectives avec l'extension du savoir à d'autres contextes.

Il est important de souligner que ces quatre qualités d'intériorisation du savoir ne suivent pas nécessairement une progression linéaire. Il peut en effet y avoir subjectivation avant qu'il n'y ait appropriation du savoir (Gregg \& Carver Sekeres, 2008; Iran-Nejad, Marsh, \& Clements, 1992). Le contrôle de la régulation semble ainsi différer selon le degré d'intériorisation du savoir. Ces éléments, ainsi que nos propres inférences sur les sources de contrôle des régulations (Buysse, 2008) et sur les indicateurs de développement (Buysse \& Vanhulle, 2009), nous conduisent à concevoir les régulations en tentant compte de leurs sources de contrôle et de leur caractère développemental. Il convient de considérer que le contrôle automatisé peut toujours être présent pour les opérations touchant à des régulations ou des parties d'une régulation routinières.

En l'état actuel de notre réflexion, les sources de contrôle des régulations lors de l'intériorisation d'un savoir se déclinent de la manière suivante (tableau 2):

1. Acquisition du savoir, apprentissage et tâches vues comme non-problématiques: a. dans les cas où l'apprenant est intéressé par le savoir: principalement, régulation sous contrôle actif de l'apprenant dans la mise en route de la régulation, avec délégation de l'ensemble ou de grandes parties de la régulation à un sous-système de régulation sous contrôle automatique; dans le cas des apprentissages scolaires, il y a une présence de contrôle 
externe, plus ou moins explicite selon les pédagogies en œuvre et les didactiques impliquées;

b. dans les cas où l'apprenant n'est pas intéressé ou quand il est démotivé: principalement, régulation sous contrôle direct du formateur ou des instruments; avec délégation de l'ensemble ou de grandes parties de la régulation à un sous-système de régulation sous contrôle automatique. ( "Je fais ce qu'il demande comme il me le dit, mais tel ou tel aspect je le fais comme je l'ai toujours fait »).

2. Appropriation du savoir, effort de compréhension en fonction des concepts fournis, tâches complexes vues comme problématiques: régulation active sous contrôle explicite de l'apprenant; avec délégation des parties routinières de la tâche ou des éléments déjà maîtrisés du savoir à des régulations sous contrôle automatique; et, dans les cas complexes, délégation volontaire ou non de parties de la régulation à des sous-systèmes sous contrôle dynamique; en situation scolaire, présence de contrôles externes, principalement de l'enseignant, surtout au niveau de la détermination de l'objectif ou de la représentation du but à atteindre par le sujet ainsi qu'au niveau de l'évaluation de la pertinence des régulations entreprises.

3. Subjectivation, compréhension au niveau personnel, recherche de sens: parfois d'abord régulation active sous contrôle explicite de l'apprenant, mais généralement dépendant d'un contrôle dynamique partiel des régulations voire d'une délégation complète de la régulation à des sous-systèmes; évaluation, feedback et ajustement sous contrôle actif de l'apprenant.

4. Maîtrise, restructuration développementale: complètement hors contrôle attentionnel, contrôle dynamique des régulations déléguées à des sous-systèmes; possible délégation volontaire et possible contrôle actif de l'évaluation et de la confirmation de l'aboutissement de ces régulations.

C'est ainsi que l'intériorisation peut être contrôlée par différentes sources influençant différentes opérations de régulation dont dépendent les conséquences développementales de l'apprentissage (voir tableau 2). 
Tableau 2: Conceptualisation du contrôle des régulations lors de l'intériorisation du savoir

\begin{tabular}{|c|c|c|c|c|c|}
\hline \multirow{2}{*}{$\begin{array}{l}\text { Apprentissage/ } \\
\text { développement }\end{array}$} & \multirow{2}{*}{$\begin{array}{l}\text { Processus composant les } \\
\text { opérations de régulation }\end{array}$} & \multicolumn{2}{|c|}{ Source de contrôle } & \multirow[b]{2}{*}{$\begin{array}{l}\text { Contrôle } \\
\text { dynamique } \\
\text { (non-atten- } \\
\text { tionnel) }\end{array}$} & \multirow[b]{2}{*}{$\begin{array}{l}\text { Qualité } \\
\text { de l'équi- } \\
\text { libration/ } \\
\text { localisation } \\
\text { principale }\end{array}$} \\
\hline & & $\begin{array}{l}\text { Contrôle } \\
\text { direct } \\
\text { (externe) }\end{array}$ & $\begin{array}{l}\text { Contrôle } \\
\text { actif } \\
\text { (atten- } \\
\text { tionnel) }\end{array}$ & & \\
\hline \multirow{6}{*}{$\begin{array}{l}\text { Acquisition } \\
\text { du savoir, } \\
\text { apprendre }\end{array}$} & $\begin{array}{l}\text { Objectif, représentation } \\
\text { initiale du but }\end{array}$ & OUI & Possible & -- & \multirow{6}{*}{$\begin{array}{l}\text { Homéosta- } \\
\text { tique localisée } \\
\text { / cortex } \\
\text { frontal }\end{array}$} \\
\hline & $\begin{array}{l}\text { Cadre (action, conception } \\
\text { ou se positionner) }\end{array}$ & OUI & Possible & Possible & \\
\hline & Orientation (comment) & OUI & OUI & -- & \\
\hline & Contrôle progression & OUI & OUI & -- & \\
\hline & Évaluation et feedback & OUI & OUI & -- & \\
\hline & Ajustement/ confirmation & OUI & OUI & -- & \\
\hline \multirow[t]{6}{*}{$\begin{array}{l}\text { Appropriation, } \\
\text { comprendre }\end{array}$} & $\begin{array}{l}\text { Objectif, représentation } \\
\text { initiale du but }\end{array}$ & OUI & OUI & Possible & \multirow{6}{*}{$\begin{array}{l}\text { Homéorhé- } \\
\text { sique localisée } \\
\text { /cortex frontal }\end{array}$} \\
\hline & $\begin{array}{l}\text { Cadre (action, conception } \\
\text { ou se positionner) }\end{array}$ & OUI & OUI & Possible & \\
\hline & Orientation (comment) & Possible & OUI & OUI & \\
\hline & Contrôle progression & Possible & OUI & OUI & \\
\hline & Évaluation et feedback & OUI & OUI & -- & \\
\hline & Ajustement/ confirmation & OUI & OUI & -- & \\
\hline \multirow{6}{*}{$\begin{array}{l}\text { Subjectivation, } \\
\text { comprendre } \\
\text { au niveau } \\
\text { personnel, } \\
\text { recherche de } \\
\text { sens }\end{array}$} & $\begin{array}{l}\text { Objectif, représentation } \\
\text { initiale du but }\end{array}$ & Possible & OUI & OUI & \multirow{6}{*}{$\begin{array}{l}\text { Homéorhé- } \\
\text { sique large } \\
\text { et diffuse / } \\
\text { pariétal-tem- } \\
\text { poral }\end{array}$} \\
\hline & $\begin{array}{l}\text { Cadre (action, conception } \\
\text { ou se positionner) }\end{array}$ & Possible & OUI & OUI & \\
\hline & Orientation (comment) & Possible & Possible & OUI & \\
\hline & Contrôle progression & -- & Possible & OUI & \\
\hline & Évaluation et feedback & -- & OUI & Possible & \\
\hline & Ajustement/ confirmation & -- & OUI & Possible & \\
\hline \multirow{6}{*}{$\begin{array}{l}\text { Maîtrise, } \\
\text { restructuration } \\
\text { développe- } \\
\text { mentale }\end{array}$} & $\begin{array}{l}\text { Objectif, représentation } \\
\text { initiale du but }\end{array}$ & -- & Possible & OUI & \multirow{6}{*}{$\begin{array}{l}\text { Homéorhé- } \\
\text { sique diffuse } \\
\text { / parié- } \\
\text { tal-frontal }\end{array}$} \\
\hline & $\begin{array}{l}\text { Cadre (action, conception } \\
\text { ou se positionner) }\end{array}$ & -- & -- & OUI & \\
\hline & Orientation (comment) & -- & -- & OUI & \\
\hline & Contrôle progression & -- & -- & OUI & \\
\hline & Évaluation et feedback & -- & Possible & OUI & \\
\hline & Ajustement/ confirmation & -- & Possible & OUI & \\
\hline
\end{tabular}


En contexte scolaire, il faut ajouter que dans presque toutes les opérations de régulation, une part plus ou moins grande de contrôle externe a lieu. Ce contrôle peut avoir son origine dans la culture de l'apprenant, dans les contraintes de la tâche, dans les objectifs acceptables, les points contrôlés habituellement ou, finalement, dans l'intervention d'un formateur. Ce contrôle d'origine culturelle peut découler des outils et/ou plus directement des signes que le contexte tient à disposition de l'apprenant.

Le contrôle des processus composant les régulations par l'apprenant s'exerce dans un réseau d'interactions résultant: du contrôle par l'apprenant lui-même; du contrôle par le formateur à travers des interventions plus ou moins explicites; du contrôle exercé par les autres apprenants sur leurs propres régulations mais aussi dans l'influence qu'ils exercent volontairement sur celles d'apprenant; du contrôle exercé ou limité par les outils et signes proposés par le contexte d'apprentissage. C'est des tensions résultant de ces interactions que se dessinera le potentiel de développement des capacités d'apprentissage autorégulé de l'apprenant (Buysse, 2012).

Il conviendrait toutefois de tenir compte de la nécessité développementale de favoriser un contrôle dynamique des autorégulations. À cela s'ajoute que, particulièrement chez le jeune enfant, la progression de l'intériorisation n'est pas linéaire comme elle est habituellement conçue dans les dispositifs didactiques, mais commence bel et bien par une subjectivation. Celle-ci repose alors principalement sur un contrôle dynamique des régulations, plutôt que sur le contrôle actif qui semble largement sollicité dans de nombreuses pratiques pédagogiques actuelles.

\section{Discussion}

Cette conceptualisation permettrait d'ouvrir la voie à un lien entre les approches cognitives et la conception des deux lignes de développement, naturelle et culturelle, chez Vygotski. Les régulations dynamiques seraient en lien avec la ligne naturelle de développement - physiologique ou biologique - et les régulations actives la ligne culturelle du développement. Ceci permettrait d'aborder entre autre certaines déficiences dans les apprentissages comme des formes différentes de préférences de contrôle de régulation et d'intégrer que la ligne biologique du développement puisse influencer les apports culturels tout autant que l'inverse (Daniels \& Hedegaard, 2011; Vygotsky, 1993).

Nous faisons l'hypothèse que la régulation dynamique est le moteur de l'équilibration générale des systèmes entre eux. Sa dominance chez les enfants en bas âge permet à notre avis de comprendre la vision globalisante qu'ont ceux-ci.

Nous pouvons aussi voir que la reconceptualisation des apprentissages sous contrôle dynamique - élaborant a posteriori des liens entre ce que le sujet apprend et d'autres connaissances - serait également ce qui permet au sujet d'utiliser dans 
d'autres contextes ce qui est appris dans un champ de connaissance particulier. Sa diminution progressive au profit de la régulation active, surtout dans le cadre de certaines formations, pourrait aisément fournir une piste d'explication pour le développement séparé des champs conceptuels. D’autres formations, qui laissent une plus large place au contrôle dynamique, notamment dans les arts à travers l'appel constant à la créativité, pourraient favoriser des transferts entre champs conceptuels.

Toutefois, la plupart des démarches didactiques s'entendent pour exiger de la part des élèves une concentration constante lors de la pratique en classe et une explicitation de leurs démarches ainsi qu'une exercisation immédiatement après la transmission du savoir. La prise de conscience par le formateur du contrôle différencié qui peut s'exercer sur les opérations de régulation pourrait amener à développer d'autres manières d'étayer les apprentissages. Par exemple, le contrôle actif et le contrôle direct pourraient être favorisés lors de la fixation des objectifs, la préparation et l'évaluation finale, alors que le contrôle dynamique pourrait être valorisé lors de l'orientation et du monitoring.

Il conviendrait donc d'intégrer des éléments pédagogiques provenant de l'approche biofonctionnelle (Gregg \& Carver Sekeres, 2008; Iran-Nejad et al., 1992; Iran-Nejad \& Zengaro, 2013) aux différentes méthodes existantes, afin de favoriser le développement de l'élève et la préservation de sa capacité créatrice. Notre conceptualisation des régulations tenant compte des sources de contrôle peut être utile dans la gestion de l'interaction, dans le coaching dans la formation professionnelle et dans une prise de conscience des effets de l'intervention de l'enseignant.

Nous pensons avoir commencé ici à tracer un lien entre les recherches en neurosciences et les travaux sur la cognition et les régulations, ainsi que proposé des pistes de prise en compte de celles-ci dans l'enseignement. Des études plus approfondies de situations d'enseignement/apprentissage seraient nécessaires pour pouvoir éclairer les effets de différentes approches pédagogiques en fonction de leur appel aux sources de contrôle des régulations.

\section{Notes}

1 En tant qu'exploration théorique, ces développements sont à notre avis applicables à toute situation de formation, même si nous mettons l'accent sur l'enseignement en contexte scolaire. Les termes formateur et apprenant sont donc utilisés la plupart du temps afin de marquer cette généralisation possible à de multiples contextes de formation, tandis que les termes enseignant et élève renvoient à des exemples de pratiques en contexte scolaire.

2 Ce qui correspond à poser le problème quant à ce qui fonde les notions (voir Dewey, 1910/1997).

3 «Un schème est la structure ou l'organisation des actions telles qu'elles se transferent ou se généralisent lors de la répétition de cette action en des circonstances semblables ou analogues» (Piaget \& Inhelder, 2004, p. 16).

4 De même, le sujet tentera d'appliquer une certaine procédure, en tant que raisonnement, à un nouveau savoir particulier à intérioriser. En effet, un savoir peut être considéré comme faisant l'objet d'une action de la part du sujet lorsqu'il tente de l'intérioriser. Cette 
procédure appliquée à un savoir donné peut être vue comme étant fondée sur un schème général de pensée (voir Buysse, 2011b, 2012).

5 Nous pouvons comprendre ici que ce sont les régulations qui permettent aux structures cognitives qui caractérisent des classes d'action équivalentes de s'actualiser et de ne pas être limitées à une reproduction du préalablement assimilé.

6 Nous introduisons cette opération de détermination afin de clairement distinguer la composante de fixation d'objectif des autres composantes de l'anticipation. En effet, l'apprentissage autorégulé, qui se définit notamment par la capacité de se fixer des objectifs sans nécessairement anticiper l'action à entreprendre (Boekaerts, 1998; Zimmerman \& Schunk, 2011), entraine, de notre point de vue, la nécessité de conceptualiser différemment cette opération.

7 Toutefois, un entrainement spécifique permettrait de réduire la surcharge cognitive liée au partage du contrôle attentionnel, mais nous considérons que cela ne s'applique pas aux conditions qui prévalent dans des contextes de formation.

8 Nous conceptualisons le fonctionnement cérébral sous une forme systémique vu la sollicitation de différentes zones du cerveau par tout processus, même au niveau du contrôle attentionnel qui ne semble pas pouvoir être localisé en un seul endroit et pour lequel les zones sollicitées varient partiellement selon à quoi l'attention est dédiée.

9 Iran-Nejad parle notamment de " mind and brain " pour illustrer ses propos. Ceci pourrait se rendre par " esprit et cerveau " et reflète l'existence d'une relation entre le contrôle attentionnel central, vu comme centre des décisions conscientes, et le reste du cerveau dans son ensemble.

10 Le contrôle - qu'il soit actif, dynamique, automatisé ou externe - désigne la source du contrôle - en anglais locus of control - exercé sur la régulation; la régulation active, dynamique, automatisée ou externe désigne, chez Iran-Nejad, la régulation qui s'est déroulée majoritairement sous cette source de contrôle.

11 Vygotski utilise bien entendu " conscients".

12 Terme de la psychologie de la créativité remontant à Wallas (1945).

13 Nous parlons d'effort heuristique lorsqu'il y a mobilisation des ressources cognitives en vue d'une découverte, d'une invention.

14 Voies axonales cortico-corticales: liaisons neuronales entre différentes parties du cortex.

15 Nous menons actuellement une recherche sur cette tension entre les besoins d'information et de contrôle du formateur et les besoins inhérents à l'intériorisation par l'apprenant. Certains indicateurs laissent paraitre que l'appel constant au contrôle actif de l'apprenant sans prise en compte de ses besoins d'avoir recours à un contrôle dynamique défavorise les apprenants souffrant de divers troubles de l'attention.

\section{Références bibliographiques}

Allal, L. (2007). Régulations des apprentissages: orientations conceptuelles pour la recherche et la pratique en éducation. In L. Allal \& L. Mottier Lopez (Éd.), Régulations des apprentissages en situation scolaire et formation (pp. 7-23). Bruxelles: De Boeck Université.

Allal, L. \& Mottier Lopez, L. (Éd.). (2007). Régulations des apprentissages en situation scolaire et formation. Bruxelles, Belgique: De Boeck Université.

Allal, L., Rouiller, Y. \& Saada-Robert, M. (1995). Autorégulation en production textuelle: observation de quatre élèves de 12 ans. Cahiers d'acquisition et de pathologie du langage (13), 17-35.

Allal, L., \& Saada-Robert, M. (1992). La métacognition: cadre conceptuel pour l'étude des régulations en situation scolaire. Archives de Psychologie (60), 265-296.

Boden, M.A. (2004). The creative mind, myths and mechanisms. London, UK: Routledge. 
Boekaerts, M. (1998). Boosting students' capacity to promote their own learning: a goal theory perspective. Learning and Instruction, 8 (1), 13-22.

Buysse, A.A.J. (2008). La médiation exercée par le formateur de théâtre sur la régulation dynamique des apprenants. In L. Mottier Lopez, Y.-E. Dizerens, G. Marcoux \& A. Perréard Vité (Éd.), Actes du 20e colloque ADMEE-Europe. Université de Genève, Suisse.

Buysse, A.A.J. (2009). Médiations contrôlantes et structurantes: une base pour penser la formation. Revue suisse des sciences de l'éducation, 31, (3), 585-602.

Buysse, A.A.J. (2011a). Les mécanismes inductifs et déductifs dans la genèse des savoirs des enseignants en formation. In J. Clénet, P. Maubant \& D. Poisson (Éd.), Débat sur la professionnalisation des enseignants: Les apports de la formation des adultes (pp. 267-307). Québec, QC: Presses de l'Université du Québec.

Buysse, A.A.J. (2011b). Une modélisation des régulations et de la médiation dans la construction des savoirs professionnels des enseignants. In P. Maubant \& S. Martineau (Éd.), Fondements des pratiques professionnelles des enseignants (pp. 243-284). Ottawa, ON: Presses universitaires d'Ottawa.

Buysse, A.A.J. (2012). Médiation des formes culturelles sur les régulations et le développement. Thèse de Doctorat, Université de Genève, http://archive-ouverte.unige.ch/unige:19382.

Buysse, A.A.J. \& Vanhulle, S. (2009). Écriture réflexive et développement professionnel: quels indicateurs? Questions Vives, 5, (11), 225-245.

Cohen, J. D., \& Schooler, J. W. (Ed.). (2013). Scientific Approaches to Consciousness. New York, NY: Psychology Press.

Daniels, H. \& Hedegaard, M. (Ed.). (2011). Vygotsky and Special Needs Education: Rethinking Support for Children and Schools. London, UK: Continuum International Publishing Group.

Dewey, J. (1910/1997). How we think. Mineola, N.Y.: Dover Publications.

Fasko, D., Jr. (2001). Education and creativity. Creativity Research Journal, 13 (3-4), 317-327.

Focant, J. (2003). Impact des capacités d'autorégulation en résolution de problèmes chez les enfants de 10 ans. Éducation et francophonie, 31, (2).

Gopnik, A. (2010). Le bébé philosophe. Paris, France: le Pommier.

Gregg, M. \& Carver Sekeres, D. (2008). Biofunctional Cognitive Theory in Poetry Instruction: Lessons from Third Grade. Research in the Schools, 15 (2), 36-54.

Hagmann, P., Cammoun, L., Gigandet, X., Meuli, R., Honey, C. J., Wedeen, V. J. \& Sporns, O. (2008). Mapping the structural core of human cerebral cortex. PLoS Biol, 6 (7: e159), 1479-1493.

Iran-Nejad, A. (1990). Active and Dynamic Self-Regulation of Learning Processes. Review of Educational Research, 60 (4), 573-602.

Iran-Nejad, A. \& Chissom, B. S. (1992). Contributions of active and dynamic selfregulation to learning. Innovative Higher Education (17), 125-136.

Iran-Nejad, A., Marsh, G. E. \& Clements, A. C. (1992). The Figure and the Ground of Constructive Brain Functionning: Brain Explicit Memory Processes. Educational Psychologist, 7 (4), 473-492.

Iran-Nejad, A. \& Zengaro, S. A. (2013). Opportunity prioritization, biofunctional simultaneity, and psychological mutual exclusion. Behavioral and Brain Sciences (36), 696-697.

Kurzban, R., Duckworth, A., Kable, J. W. \& Myers, J. (2013). An opportunity cost model of subjective effort and task performance. Behavioral and Brain Sciences (36), 661-726.

Lubart, T. \& Georgsdottir, A. S. (2004). Créativité, haut potentiel et talent. Psychologie française (49), 277-2919.

Piaget, J. (1974). La prise de conscience. Paris, France: Presses Universitaires de France.

Piaget, J. (1975). L'équilibration des structures cognitives: problème central du développement. Paris, France: PUF. 
Piaget, J. (1977). Recherches sur l'abstraction réfléchissante (Vol. 1 et 2). Paris, France: Presses Universitaires de France.

Piaget, J., \& Inhelder, B. (2004). La psychologie de l'enfant. Paris, France: PUF.

Runco, M. A. (2003). Creativity, cognition, and their educational implications. In J. Houtz (Ed.), The educational psychology of creativity. Perspectives on creativity (pp. 25-56). Cresskill, NJ: Hampton Press, Inc.

Saada-Robert, M. (1992). La construction microgénétique d'un schème élémentaire Le cheminement des découvertes de l'enfant (pp. 119-137). Neuchâtel: Delachaux et Niestlé.

Saada-Robert, M., \& Brun, J. (1996). Les transformations des savoirs scolaires: apports et prolongements de la psychologie génétique. Perspectives, 26,(1), 25-37.

Saada-Robert, M. (1998). Situations didactiques complexes et acquisition intégrée de connaissances spécifiques: le cas de l'orthographe en production textuelle. In M. Brossard \& J. Fijalkow (Éd.), Apprendre à l'école: perspectives piagétiennes et vygotskiennes (pp. 81-101). Bordeaux, France: Presses universitaires de Bordeaux.

Saada-Robert, M. (2012). From individual to didactic microgenesis: Studies on situated knowledge transformations. In E. Marti \& C. Rodriguez (Ed.), After Piaget (pp. 187-206). New Brunswick, NJ: Transactions Publishers.

Saada-Robert, M. \& Brun, J. (1996). Les transformations des savoirs scolaires: apports et prolongements de la psychologie génétique. Perspectives, 26, (1), 25-37.

Sawyer, R. K. (2012). Explaining creativity: the science of human innovation. Oxford, UK: Oxford University Press.

Sawyer, R. K., John-Steiner, V., Moran, S., Sternberg, R. J., Feldman, D. H., Nakamura, J. \& Csikszentmihalyi, M. (2003). Creativity and development. New York, NY: Oxford University Press.

Shapiro, S. R. \& Livingston, J. A. (2000). Dynamic Self-Regulation: The Driving Force Behind Academic Achievement. Innovative Higher Education, 25(1).

Soon, C. S., Brass, M., Heinze, H.-J. \& Haynes, J.-D. (2008). Unconscious determinants of free decisions in the human brain. Nature Neuroscience, 11 (5), 543-545.

Valsiner, J. (1997). Culture and the Development of Children'Action: A Theory of Human Development (2e ed.). New York, NY: John Wiley \& Sons

Vartanian, O. (2013). Fostering Creativity: Insights from Neuroscience. In O. Vartanian, A. S. Bristol \& J. C. Kaufman (Ed.), Neuroscience of Creativity (pp. 257-272). Cambridge, MA: MIT Press.

Vohs, K. D., Baumeister, R. F., Schmeichel, B. J., Twenge, J. M., Nelson, N. M., \& Tice, D. M. (2014). Making choices impairs subsequent self-control: A limited-resource account of decision making, self-regulation, and active initiative. Motivation Science, 1 (S), 19-42.

Volet, S. \& Vauras, M. (Ed.). (2013). Interpersonal Regulation of Learning and Motivation. New York, NY: Routledge.

Vygotski, L. (1925/2005). Psychologie de l'art. Paris: La Dispute.

Vygotsky, L. S. (1993). The Collected Works of L.S. Vygotsky (vol. 2, The Fundamentals of Defectology: Abnormal Psychology and Learning Disabilities) (J. E. Knox \& C. B. Stevens, trad.). New York, NY: Plenum Press.

Wallas, G. (1945). The art of Thought. London, UK: Watts.

Yeung, N., Nystrom, L. E., Aronson, J. A., \& Cohen, J. D. (2006). Between-Task Competition and Cognitive Control in Task Switching. The Journal of Neuroscience, 26 (5), 1429-1438.

Zimmerman, B. J., \& Schunk, D. H. (Ed.). (2011). Handbook of Self-Regulation and Performance. New York, NY: Routledge.

Mots-clés: Contrôle attentionnel, régulation, processus, apprentissage, développement 


\section{Aufmerksamkeitskontrolle und Lernen. Ein anderer Weg, um die erforderliche Aufmerksamkeit während des Lern- prozesses zu gestalten}

\section{Zusammenfassung}

Zahlreiche Arbeiten betonen die kognitiven Arbeitsprozesse, die abseits der Aufmerksamkeitskontrolle durch die Lernenden ablaufen und dies besonders in einer Entwicklungsperspektive. Die Regulierungsprozesse, deren sich die Ler-nenden nicht bewusst sind, scheinen eine wichtige Rolle zu spielen, was das Verstehen und das Herstellen von Beziehungen mit dem zuvor angeeigneten Stoff angeht. Ihnen kommt auch eine wesentliche Rolle beim Problemlösen zu. Trotzdem verlangt die Mehrheit der didaktischen Vorgehensweisen von den Schülerinnen und Schülern, sich während Aktivitäten in der Klasse zu konzent-rieren und ihr Vorgehen jeweils explizit zu machen, ebenso wie sie eine Umset-zung unmittelbar nach dem Wissenstransfer erfordern. Aufgrund unserer Stu-dien schlagen wir eine Konzeptualisierung der Verbindungen zwischen den Re-gulationen und deren Kontrollquellen vor, die bestimmte bekannte Elemente der Funktionsweise des Gehirns berücksichtigen. Wir greifen auch die Komplexität der Wechselwirkungen zwischen den unterschiedlichen Kontrollquellen im Rahmen von Aktivitäten in der Klasse auf.

Schlagworte: Aufmerksamkeitskontrolle, Regulationsprozesse, Lernprozesse, Entwicklung

\section{Controllo dell'attenzione durante l'apprendimento Un altro modo di concepire l'attenzione dello studente durante i processi di regolazione}

\section{Riassunto}

Numerosi lavori mostrano il lavoro cognitivo che si svolge al di fuori del controllo dell'attenzione dello studente, in particolare da un punto di vista dello sviluppo. Le regolazioni di cui lo studente non ha coscienza sembrano avere un ruolo importante nella comprensione e nei legami con la materia precedentemente appresa. Hanno anche un ruolo fondamentale nella risoluzione di problemi. Tuttavia, la maggior parte degli approcci didattici esigono da parte degli allievi una concentrazione durante la pratica in classe e una spiegazione del loro approccio, così come un'azione pratica subito dopo la trasmissione del sapere. Sulla base delle nostre ricerche, proponiamo una concettualizzazione dei legami fra la regolazione e le fonti di controllo tenendo conto di certi elementi noti sul funzionamento del cervello. Affrontiamo inoltre la complessità delle interazioni fra le differenti fonti di controllo nell'ambito del lavoro in classe. 
Parole chiave: controllo dell'attenzione, regolazione, procedura, apprendimento, sviluppo

\title{
Attentional control and learning Another way to look at attention required during learning
}

\begin{abstract}
Summary
Recent research has stressed the cognitive processes that are not under the attentional control of the learner, especially from a developmental point of view. The regulatory processes that take place without the learner's conscious knowledge seem to play an important part in his comprehension and in his ability to link newly acquired notions with already mastered concepts. These unconscious regulatory processes are also crucial to problem solving. However, most approaches to teaching require students to focus their attention during classroom practices and ask them to make their solving procedures explicit, as well as practice immediately after knowledge transfer. Based on our researches, we offer a conceptualization of the relations between regulatory processes and locus of control of regulatory processes. We aim at taking into account a number of known facts about the functioning of the brain and the complexity of the interactions between different loci of control occurring during teaching.
\end{abstract}

Keywords: Attentional control, regulatory processes, learning, processes, development 